\title{
Large malignant ovarian tumors during pregnancy: two cases
}

This article was published in the following Dove Press journal:

OncoTargets and Therapy

17 November 2014

Number of times this article has been viewed

\section{Dong Xu \\ Cheng Liang \\ Jing $\mathrm{He}$}

Gynecological and Obstetrical Department, Women's Hospital, School of Medicine, Zhejiang University, Hangzhou, People's Republic of China
Correspondence: Jing He Gynecological and Obstetrical Department, Women's Hospital, School of Medicine, Zhejiang University, I Xueshi Road, Hangzhou, 310006 , People's Republic of China Email hejing_zju@।63.com
Abstract: The present study reports two cases of large ovarian malignancy during pregnancy, which is very rare. The two patients were received between Nov 2012 and Feb 2013 at Gynecological and Obstetrical Department, Women's Hospital, School of Medicine, Zhejiang University (People's Republic of China). Both cases present tumor sized more than $20 \mathrm{~cm}$, with one case of $40 \mathrm{~cm}$. Both patients underwent timely cesarean section, with survival of the Child, and successful removal of the tumor. All patients showed good outcome in the follow-up period. Therefore the large ovarian malignancy during pregnancy could be well treated after careful clinical evaluation.

Keywords: ovarian malignancy, pregnancy, incidence

\section{Introduction}

Ovarian malignancies during pregnancy are rare. ${ }^{1-7}$ In a large survey in Canada, the incidence of malignant ovarian tumors during pregnancy was 0.0416 per 1,000 pregnancies, comprising mainly borderline tumors followed by epithelial tumors. ${ }^{4,8}$ In Europe and the USA, the incidence was $0.04-0.11$ per 1,000 pregnancies, and comprised mostly borderline tumors. ${ }^{4}$ In Asia, the incidence was 0.106 per 1,000 pregnancies, with $40 \%$ being borderline tumors. ${ }^{3}$ It was also found that grade I tumors accounted for up to $62.5 \%$ of all cases, and histological type was mainly low-grade malignancy $(62.5 \%)$. Borderline tumors accounted for up to $50 \%$ of these cases. ${ }^{4,5,8-10}$ Previously reported cases have had tumor diameters in the range of 5-24 cm. Here we present two rare cases of large ovarian malignancy during pregnancy, both involving tumors with diameters larger than $20 \mathrm{~cm}$, with one reaching $40 \mathrm{~cm}$.

\section{Case report I}

Our first case was a 24-year-old married woman who was admitted to our hospital on February 4, 2013, due to 35 weeks of menopause, and detection of pelvic mass for 4 months. The first ultrasound examination on September 25, 2012 indicated an intrauterine pregnancy with a single live embryo and a large $20-30 \mathrm{~cm}$ abdominal cystic mass. A second ultrasound examination on November 7, 2012 showed the mass to be more than $30 \mathrm{~cm}$ in diameter. The patient reported no specific symptoms and was not receiving any medication.

On admission to our hospital, general examination revealed a body temperature of $37.4^{\circ} \mathrm{C}$, a heart rate of 105 beats per minute, a respiratory rate of 22 per minute, a blood pressure of 140/100 $\mathrm{mmHg}$, and a bulging belly shape. Her fundal height was $52 \mathrm{~cm}$ and her abdominal circumference was $120 \mathrm{~cm}$. The patient had occasional 
contractions, but vaginal examination showed that the cervix was not open. Ultrasound examination on February 4, 2013 showed the fetus to be in the left occiput anterior position, a fetal heart rate of 124 beats per minute, fetal movement, a femur length of $6.1 \mathrm{~cm}$, an anterior placenta grade $\mathrm{I}+$, an amniotic fluid level of $3 \mathrm{~cm}$, and an umbilical artery S/D ratio of 3.0. The abdominal cavity was filled with plenty of fluid, measuring $25.4 \mathrm{~cm}$ at its deepest point. A substantial $11.2 \times 11.6 \times 10.4 \mathrm{~cm}$ cauliflower-shaped mass was detectable, with little blood flow in the vein blood flow. A $5.6 \times 4.5 \times 5.6$ cm cystic mass was present to the right of the uterus, showing separation inside and clean solution inside. Urinalysis revealed proteinuria ++ and blood investigations revealed total protein $53.7 \mathrm{~g} / \mathrm{L}$, albumin $27.1 \mathrm{~g} / \mathrm{L}$, C-reactive protein $31.2 \mathrm{mg} / \mathrm{L}$, aspartate aminotransferase $84 \mathrm{U} / \mathrm{L}$, alanine aminotransferase $43 \mathrm{U} / \mathrm{L}$, and tumor markers (cancer antigen 1.1 ng/mL, alpha-fetoprotein $168.8 \mathrm{ng} / \mathrm{mL}, \mathrm{CA}-125$ 50.9 U/mL), with no other obvious abnormalities.

The patient was diagnosed as being at 35 weeks' gestation with a left occiput anterior pregnancy, possible fetal distress, severe pre-eclampsia, and an abdominal mass to be determined (query ovarian tumor). The patient was started on oral labetalol $100 \mathrm{mg}$ at 12-hour intervals to decrease her blood pressure, intravenous magnesium sulfate $40 \mathrm{~mL}$ as an antispasmodic, $100 \mathrm{~mL}$ of intravenous albumin as a protein supplement, and a $40 \mathrm{~mL}$ injection of compound glycyrrhizin for liver protection. After consultation with doctors from the departments of medicine and oncology, and obtaining approval from the department of critical care, the patient underwent lower uterine segment cesarean section, laparotomy, and fertility-preserving ovarian cancer resection (right adnexectomy, greater omentum resection, pelvic lymph node dissection, appendectomy, left ovary necropsy) under epidural anesthesia. During surgery, $50 \mathrm{~mL}$ of free, clear-colored, pelvic fluid was taken for cytology examination.

The uterus was in the anterior position, with a size indicating 8 months' gestation. A lower uterine segment cesarean section was performed successfully, with head position delivery of a live female baby weighing 2,750 $\mathrm{g}$ with an Apgar score of 9 at one and 5 minutes. The uterine wall showed abnormal contraction, which resolved following hemabate injection. After the surgery, we found that the uterine surface was smooth, with a rectal fossa uterus and bladder peritoneal fold back membrane, and a normal left ovary with a smooth surface showing no adhesion to surrounding tissues. The left fallopian tube showed no abnormalities, with free end visible. The right ovary was cystic and was increased in size to $45 \times 40 \times 40 \mathrm{~cm}$, with the top edge reaching the diaphragm and adhering to the greater omentum. The cystic wall was thick and unbroken. The right fallopian tube was thickened, and free end could be seen. The diaphragmatic surface was smooth, and the pelvic lymph nodes showed no abnormalities.

Right oophorectomy was then attempted; however, during surgery, the right ovarian tumor was found to be too large to expose and remove directly. Aspiration of cystic fluid revealed the polycystic nature of the tumor. During aspiration, the cyst ruptured, releasing about $15,000 \mathrm{~mL}$ of pale yellow, clear, and viscous, cystic fluid. The right accessory ovary was then removed, exposing a $45 \times 40 \times 40 \mathrm{~cm}$ mass with a smooth surface and polycystic features, and a solid $12 \times 11 \times 10 \mathrm{~cm}$ mass. Intraoperative frozen section examination revealed a local ovarian mucinous cystadenoma at the right side of the borderline. Examination of the pelvic fluid revealed red blood cells and inflammatory cells, but no tumor cells. Intraoperative discussion with the patient and family members lead to fertility-sparing radical surgery for ovarian

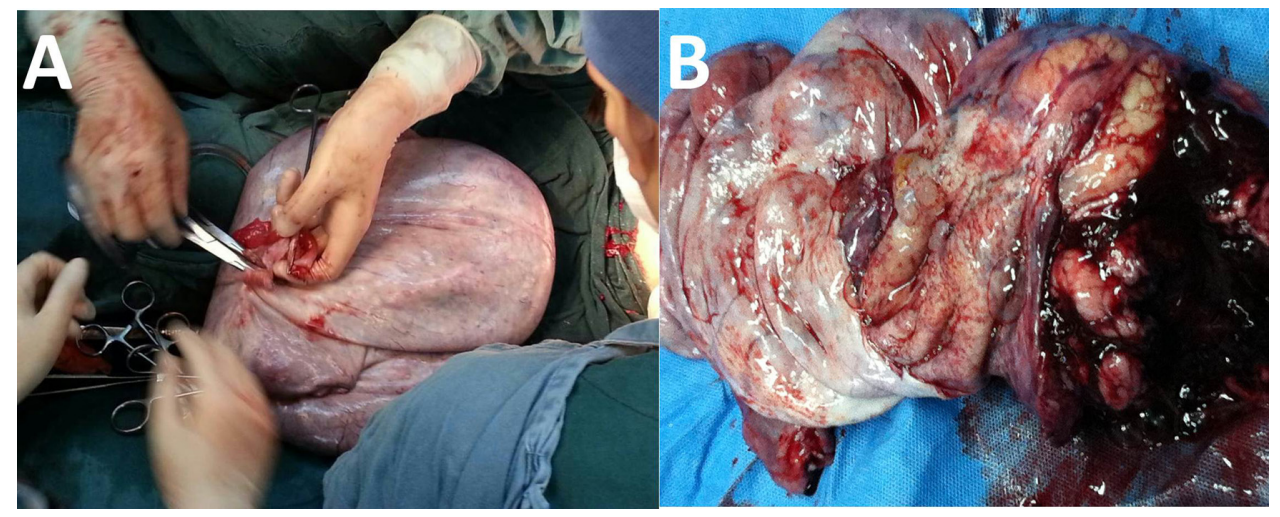

Figure I Huge ovarian tumor during pregnancy.

Notes: (A) General view of the ovarian tumor in case one (after removal of I0,000 mL of cyst fluid) during surgery. The full size of the tumor was $45 \times 40 \times 40 \mathrm{~cm}$. (B) Internal side of the cyst after opening the ovarian tumor in case two (smooth surface, multiple-room cyst. Pathological examination showed a juvenile granulosa cell tumor. 
cancer. The surgery proceeded successfully, with blood loss of $500 \mathrm{~mL}$, a steady pulse, and a clear-colored urine output of around 1,000 mL. After surgery, pathological analysis confirmed a diagnosis of right grade I borderline mucinous ovarian adenocarcinoma. The right fallopian tube tissue and blood vessels were negative. Ten surrounding lymph nodes were also negative.

Immunohistochemistry revealed CK-negative and Vimentin-positive staining. Nine days after surgery, the patient was discharged from hospital with a recommendation for chemotherapy. The patient was in a stable condition (without chemotherapy) at follow-up 3 months after surgery (Figure 1).

\section{Case report 2}

Our second case was a 24-year-old married woman who was admitted to our hospital on November 7, 2012 due to 32 weeks of menopause, and 2 days after detection of pelvic mass. Examination at 16 weeks had shown no abnormality and indicated a low-risk pregnancy. On November 5, 2012, at 32 weeks' gestation, ultrasound examination revealed a "right upper abdominal split septum-like huge cystic mass (right ovarian cyst?)". On admission to hospital, ultrasound examination revealed little amniotic fluid, a cystic mass in the right cavity, which was suspicious for ovarian cancer. The patient had gained $18 \mathrm{~kg}$ in weight during her pregnancy. Physical examination revealed a body temperature of $36.6^{\circ} \mathrm{C}$, a heart rate of 96 beats per minute, a respiratory rate of 18 per minute, blood pressure $109 / 83 \mathrm{mmHg}$, no tenderness or rebound tenderness, and a large palpable mass in the right upper quadrant. The height of the uterus was $29 \mathrm{~cm}$ and the abdominal circumference was $96 \mathrm{~cm}$, with no contraction. The fetus was in the left occiput anterior position, the fetal heart rate was 158 beats per minute, fetal movement was detectable, femur length was $6.0 \mathrm{~cm}$, Gr-level anterior placenta, the amniotic fluid index was $7.4 \mathrm{~cm}$, and the umbilical artery S/D ratio was 2.7. The huge cystic mass was in the right cavity, with top edge to the liver and bottom edge to the pelvis, the size of $28 \times 25 \times 12 \mathrm{~cm}$; it shows multiple rooms separated and rich blood flow, the cyst fluids are not in clear color. One room shows uneven echo and rich blood flow, RI: 0.2. There was some free fluid in the right lower quadrant, with a depth of $6.1 \mathrm{~cm}$. Examination of tumor markers revealed a carcinoembryonic antigen of $0.4 \mathrm{ng} / \mathrm{mL}$, an alfa-fetoprotein of $266.8 \mathrm{ng} / \mathrm{mL}$, and a CA-125 of $466.9 \mathrm{U} / \mathrm{mL}$.

On November 13, 2012, the patient underwent lower uterine segment cesarean section and fertility-preserving ovarian cancer resection (right adnexectomy, partial greater omentum resection, pelvic lymph node dissection, pelvic lymphadenectomy, peritoneal multiple biopsy) under epidural anesthesia. During surgery, $200 \mathrm{~mL}$ of clearcolored free pelvic liquid was taken and used for cytology examination.

A lower uterine segment cesarean section was performed successfully, with safe delivery of a live female baby weighing 1,800 $\mathrm{g}$ with an Apgar score of 10 at one and 5 minutes. The left ovary was of normal size, while the right ovary showed cystic enlargement to $30 \times 25 \times 15 \mathrm{~cm}$. The top edge of the right ovary reached to the diaphragm, the right reached to the colon, and the left extended to the greater curvature of the stomach. It had a smooth surface and there was no adhesion to surrounding tissues or rupture. The right fallopian tube was edematous and thickened, and free end could be seen. There were no clear abnormalities in the stomach, liver, or spleen, and the pelvic lymph nodes were normal. Aspiration of 2,000 $\mathrm{mL}$ of cystic fluid result in the exposure of the right accessory tumor, dissection of which showed a cystic mass of $30 \times 25 \times 15 \mathrm{~cm}$ with a smooth surface. Examination of pelvic fluid revealed no cancer cells. Rapid frozen section examination suggested a right ovarian juvenile granulosa cell tumor.

Routine pathological examination after surgery confirmed the right ovarian juvenile granulosa cell tumor. The right fallopian tube and blood vessels were negative, and 19 surrounding lymph nodes were also negative. On the first day after surgery, hematology revealed white cells $16.5 \times 10^{9} / \mathrm{L}$, neutrophils $66.4 \%$, and hemoglobin $94 \mathrm{~g} / \mathrm{L}$, and biochemistry showed total protein $45.2 \mathrm{~g} / \mathrm{L}$, albumin $26.4 \mathrm{~g} / \mathrm{L}, \mathrm{C}$-reactive protein $94.6 \mathrm{mg} / \mathrm{L}$, and plasma D-2 dimer $4.16 \mathrm{mg} / \mathrm{L}$. The patient was treated with anti-inflammatory drugs and symptomatic support. Six days after surgery, blood investigations revealed white blood cells of $8.0 \times 10^{9} / \mathrm{L}$, neutrophils $65.9 \%$, hemoglobin $107 \mathrm{~g} / \mathrm{L}$, C-reactive protein $27.3 \mathrm{mg} / \mathrm{L}$, and plasma D-2 dimer $2.97 \mathrm{mg} / \mathrm{L}$, along with tumor markers (alfa-fetoprotein $39.5 \mathrm{ng} / \mathrm{mL}, \mathrm{CA}-125359.9 \mathrm{U} / \mathrm{mL}, \mathrm{CA}-15332.6 \mathrm{U} / \mathrm{mL}$ ). Eight days after surgery, the patient was transferred to the department of oncology. Starting on November 23, 2012, the patient received first-line BEP (bleomycin, etoposide, diamminedichloroplatinum(II) chemotherapy) as recommended by the International Federation of Gynecology and Obstetrics for cord stromal tumors, as well as euprolide by hypodermic injection to preserve ovarian function. The second phase started on December 25, 2012, and the third phase on January 23, 2013. Follow-up after 2 years showed that they maintained the healthy state. 


\section{Discussion}

Previous reports indicate that most accessory ovarian masses are benign, and that malignant ovary tumors are rare during pregnancy. However, at our hospital, we have diagnosed two cases in the past 2 years (2012-2013).

Patients may have no symptoms and no evidence to suggest that the tumor is affecting fetal growth and development. The large size of the tumor may at some time point hinder uterus growth, leading to premature birth or miscarriage. Clinical diagnosis of ovarian tumors at an early stage relies on bimanual, triple diagnosis, or sonographic findings, although when tumor growth is rapid, it may cause bloating, abdominal pain, an abdominal mass, and marked pressure symptoms. When the tumor ruptures due to external pressure, there might be internal bleeding and symptoms of peritoneal irritation.

Pelvic ultrasound examination is the most reliable method for detection of an ovarian tumor, in terms of position, size, morphology, relationship to the uterus, with cystic fluid or not, with cyst wall separation or not and existence of pelvic fluid. Once a pelvic mass is detected, ultrasound follow-up during pregnancy helps to determine the nature of the mass. The mass should be observed dynamically under ultrasound examination. The vast majority of ovarian cysts are follicular cysts or corpus luteum cysts, with a diameter of less than $5 \mathrm{~cm}$ (very rarely reaching $11 \mathrm{~cm}$ ). Ninety percent of functional cysts disappear as the pregnancy progresses, and diminishes at 14 weeks. Six percent of cysts are less than $6 \mathrm{~cm}$, and $39 \%$ larger than $6 \mathrm{~cm}$ persist afterwards. A single cyst measuring less than $10 \mathrm{~cm}$, one side, and without ascites can be left to 18 weeks without further medication; however, if the cyst persistently increases in size, surgical exploration is required. For mixed cysts larger than $5 \mathrm{~cm}$, bilateral sides, or with nipple should be followed up with ultrasound; if it persists or the size increases by $30 \%-50 \%$, surgical exploration is required. ${ }^{2,11,12}$ It has been reported that cysts larger than $15 \mathrm{~cm}$ are 12 times more likely to be malignant than cysts less than $6 \mathrm{~cm}$ in diameter. ${ }^{13}$ In the first case presented in this report, ultrasound revealed a solid mass larger than $10 \mathrm{~cm}$, and blood flow suggested a malignant tumor. It should be noted that the ultrasound diagnosis is limited by the experience of the clinicians and sonographers, and is blocked by abdominal organs during pregnancy, which could lead to misdiagnosis. For instance, cyst fluid could have been considered to be pelvic fluid on early ultrasound examination in the first case. Therefore, routine gynecological ultrasound examination should be performed to exclude any possible mass, and be followed by magnetic resonance imaging in cases where there is diagnostic difficulty.
CA-125 is a widely used epithelial ovarian tumor marker, with an $80 \%$ positive detection rate in most ovarian serous adenocarcinomas. However, CA-125 is not specific for ovarian tumors, and its levels also increase during pregnancy. Nonpregnant patients with nonserous, mixed, or mucinous borderline ovarian tumors have been found to have CA-125 levels of $9,918 \mathrm{kU} / \mathrm{L}, 11,718 \mathrm{kU} / \mathrm{L}$, and 3,419 kU/L, respectively. ${ }^{14,15}$ In our two patients, increased CA-125 may have been of some diagnostic value, but CA-125 still lacks enough accuracy in evaluating the treatment efficiency. ${ }^{12}$

Treatment of a malignant ovarian tumor during pregnancy is not significantly different from that in nonpregnant women. The diagnosis should be followed by timely surgery, irrespective of the stage of pregnancy. An intraoperative frozen section is required to identify the tumor. For a malignant ovarian tumor during early pregnancy, abortion is required as the standard for following managements.

There are several features characteristic of malignant ovarian tumors during pregnancy: they tend to occur mainly in first-baby young mothers; tumors are mostly in an early phase; cell differentiation is mainly low-grade malignant; and the prognosis is better than in nompregnant cases. Therefore, some patients can be treated with conservative surgery: Stage Ia period of granulosa cell tumors, Stage Ia dysgerminoma, Stage Ial grade immature teratoma, Stage Ia borderline epithelial tumors, Stage Ia1 grade squamous cell carcinoma. For unilateral tumor cases, the accessory tumor can be removed and a biopsy should be performed for cytological examination. If no cancer cells are found, there is no need for chemotherapy and the pregnancy can continue. After the delivery, a second surgery is required. If there are clear symptoms of ovarian cancer, such as abdominal distension, rapid cyst growth, signs of nipples or blood flow in the cyst, or presence of ascites, surgery should be performed immediately regardless of the pregnancy. If the mother has a strong desire for the fetus to survive, conservative surgery can be performed and the second surgery can be performed after cesarean section. If the tumor grade increases, adjuvant chemotherapy or radical surgery may be required. ${ }^{16}$ For a progressive tumor, the pregnancy should be terminated before cytoreductive surgery.

For epithelial cancer, combined platinum-based chemotherapy can be used. For nonepithelial cancer, the BEP, etoposide-cisplatin, and cisplatin-vinblastine-bleomycin chemotherapy protocol shows no adverse effects on the fetus. After termination of pregnancy, combined platinum-paclitaxel therapy can be used. Intraperitoneal chemotherapy is effective after cytoreductive surgery for Phase III ovarian tumors. ${ }^{17}$ 
In summary, the treatment of a malignant ovarian tumor during pregnancy should be based on stage of pregnancy, the nature of the tumor, individual symptoms, safety of the fetus, and considering the fertility of the patient after treatment. Treatment should be given to maximize the survival rate and at the most proper time point.

\section{Acknowledgment}

The authors thank the patients for providing consent for the publication of this case series and any accompanying images.

\section{Disclosure}

The authors report no conflicts of interest in this work.

\section{References}

1. Dobashi M, Isonishi S, Morikawa A, et al. Ovarian cancer complicated by pregnancy: analysis of 10 cases. Oncol Lett. 2012;3(3):577-580.

2. Kwon YS, Mok JE, Lim KT, et al. Ovarian cancer during pregnancy: clinical and pregnancy outcome. J Korean Med Sci. 2010;25(2): 230-234.

3. Gasim T, Al Dakhiel SA, Al Ghamdi AA, et al. Ovarian tumors associated with pregnancy: a 20-year experience in a teaching hospital. Arch Gynecol Obstet. 2010;282(5):529-533.

4. Machado F, Vegas C, Leon J, et al. Ovarian cancer during pregnancy: analysis of 15 cases. Gynecol Oncol. 2007;105(2):446-450.

5. Ueda M, Ueki M. Ovarian tumors associated with pregnancy. Int $J$ Gynaecol Obstet. 1996;55(1):59-65.
6. Matsuyama T, Tsukamoto N, Matsukuma K, Kamura T, Kaku T, Saito T. Malignant ovarian tumors associated with pregnancy: report of six cases. Int J Gynaecol Obstet. 1989;28(1):61-66.

7. Halila H, Stenman UH, Seppala M. Ovarian cancer antigen CA 125 levels in pelvic inflammatory disease and pregnancy. Cancer. 1986;57(7):1327-1329.

8. Gezginc K, Karatayli R, Yazici F, Acar A, Celik C, Capar M. Ovarian cancer during pregnancy. Int J Gynaecol Obstet. 2011;115(2):140-143.

9. Tsai HJ. Metastatic bilateral malignant ovarian tumors associated with pregnancy. Taiwan J Obstet Gynecol. 2011;50(3):405-406.

10. Singhal SR, Nanda S, Chaudhry P, Sen J, Singhal SK. Metastatic bilateral malignant ovarian tumors associated with pregnancy. Taiwan J Obstet Gynecol. 2009;48(2):167-168.

11. Ngu SF, Cheung VY, Pun TC. Surgical management of adnexal masses in pregnancy. JSLS. 2014;18(1):71-75.

12. Marret $\mathrm{H}$, Lhomme $\mathrm{C}$, Lecuru F, et al. Guidelines for the management of ovarian cancer during pregnancy. Eur J Obstet Gynecol Reprod Biol. 2010;149(1):18-21.

13. Koo YJ, Kim TJ, Lee JE, et al. Risk of torsion and malignancy by adnexal mass size in pregnant women. Acta Obstet Gynecol Scand. 2011;90(4):358-361.

14. Moore RG, Miller MC, Eklund EE, Lu KH, Bast RC Jr, Lambert-Messerlian G. Serum levels of the ovarian cancer biomarker HE4 are decreased in pregnancy and increase with age. Am J Obstet Gynecol. 2012;206(4):349. e341-e347.

15. Whiteman DC, Siskind V, Purdie DM, Green AC. Timing of pregnancy and the risk of epithelial ovarian cancer. Cancer Epidemiol Biomarkers Prev. 2003;12(1):42-46.

16. Hasegawa T, Ishii $Y$, Yonezawa R, et al. Stage I ovarian cancer cases during early, mid and late pregnancy periods: three case reports and review of the literature. J Obstet Gynaecol Res. 2011;37(6):650-655.

17. Brewer M, Kueck A, Runowicz CD. Chemotherapy in pregnancy. Clin Obstet Gynecol. 2011;54(4):602-618.
OncoTargets and Therapy

\section{Publish your work in this journal}

OncoTargets and Therapy is an international, peer-reviewed, open access journal focusing on the pathological basis of all cancers, potential targets for therapy and treatment protocols employed to improve the management of cancer patients. The journal also focuses on the impact of management programs and new therapeutic agents and

\section{Dovepress}

protocols on patient perspectives such as quality of life, adherence and satisfaction. The manuscript management system is completely online and includes a very quick and fair peer-review system, which is all easy to use. Visit http://www.dovepress.com/testimonials.php to read real quotes from published authors 